\title{
Téoros
}

Revue de recherche en tourisme

\section{Tourisme dans le marais audomarois}

\section{Odile Dufly}

Volume 10, numéro 1, mars 1991

Tourisme et environnement

URI : https://id.erudit.org/iderudit/1079814ar

DOI : https://doi.org/10.7202/1079814ar

Aller au sommaire du numéro

Éditeur(s)

Université du Québec à Montréal

ISSN

0712-8657 (imprimé)

1923-2705 (numérique)

Découvrir la revue

Citer cet article

Dufly, O. (1991). Tourisme dans le marais audomarois. Téoros, 10(1), 16-20.

https://doi.org/10.7202/1079814ar d'utilisation que vous pouvez consulter en ligne.

https://apropos.erudit.org/fr/usagers/politique-dutilisation/ 


\section{Tourisme dans le marais audomarois}

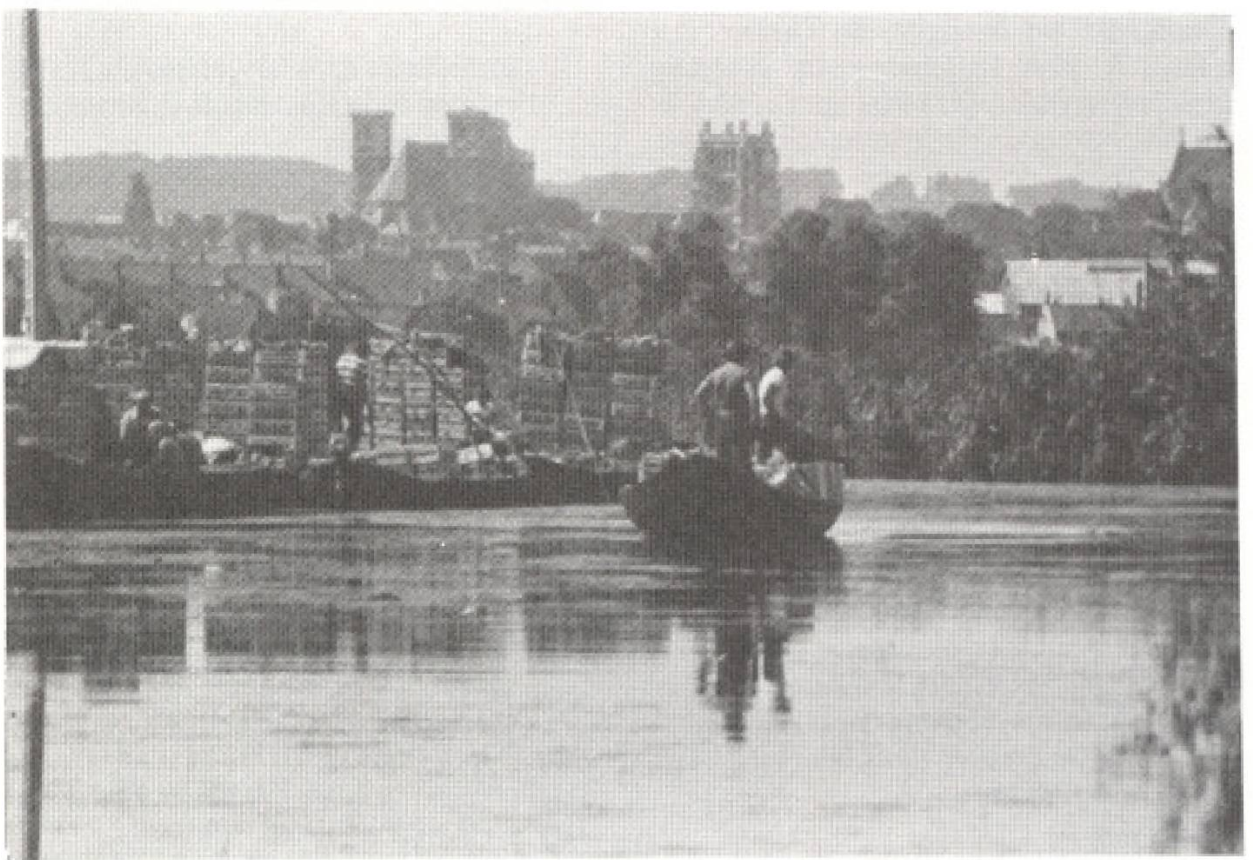

De l'autre côté de l'Atlantique, dans cette vieille Europe, un point clignote tel un phare qui veille ou qui appelle pour un audelà. Ici, la mer pénètre dans les terres comme la lumière devient feux-follets. Le monde merveilleux et étrange des marais défile devant les bélandres ${ }^{(1)}$ en commerce àSaint-Omer, cette ville flamande très florissante pendant le moyen-âge devenue française il y a près de trois siècles, "perchée dans les Hauts-de-France" ${ }^{\text {"(2) }}$. Le marais de l'audomarois est là, immobile à la porte de la ville et de son agglomération de 70000 habitants. Ses portes d'eau s'ouvrent, le temps d'un voyage à travers l'histoire, pour que s'aiguise notre regard sur les conditions de sa mise en valeur, source permanente de conflits d'intérêts.

Petit, le marais audomarois l'est; 3400 ha de terreset d'eau conquis sur la mer il y a 13 siècles, alimenté par l'Aa (mot de deux lettres bien connu des cruciverbistes). Spécifique aussi, puisque caractérisé par une présence hụmaine et une activité marâichère fortes... le seul marais de France à développer du chou-fleur. Riche par la diversité, fragile, mi-autarcique...

Odyle Dufly est chargée de Mission AménagementEnvironnement, Espace Naturel Régional (France)
Bref, un site qui vit, subit, réagit, évolue. $\mathrm{Et}_{\text {z }}$ in-fine, un site qui suscite de l'intérêt pour son devenir à l'approche de l'an 2000 auprès des décideurs, des gestionnaires, des protecteurs de l'environnement, voire de la population.

\section{Le marais audomarois et ses particularismes: richesse ou frein?}

Ce petit pays d'eau a toujours vécu replié sur lui-même.

La relation de l'homme avec son marais était une relation d'amour. L'eau était son ennemie car elle pouvait en cas de crues remettre en cause son long et pénible travail de mosaïque d'îles. Mais l'eauétait aussisa mère nourricière car cette terre fertile sortie des marécages a toujours eu besoin d'eau, encore maintenant. Le brouckailleur ou maraîcher consacrait, dans son temps de travail, un temps pour $1^{\prime}$ entretien du marais. Le curage des fossés et rivières se faisait donc l'hiver avec des outils manuels appropriés; la baguernette pour retirer la vase et l'épandre sur les terres où la grèpe pour extraire la tourbe et se chauffer. Il savait que c'était vital car la voie d'eau était aussi sa route pour aller au travail ou retourner à la maison en bacóvẹe
Sa vie était donc différente, rythmée par d'autres lois... de l'ordre particulier des us et coutumes mis en place et gêrés par une communauté solidaire autour de l'eau. Les ingrédients étaient réunis pour faire de ce pays "un isolat social à composantes fortes", selon l'expression des sociologues.

Des travaux de recherche-animation engagés dans les années 1980-1985 pour la préfiguration du Parc naturel régional-car il importait de comprendre avant d'agir ont révélé les spécificités de ce pays d'eau de culture flamande riche en traditions. Ils ont aussi souligné les phénomènes d'acculturation que la voie navigable en communication avec la mer ou le chemin de fer ont généré en entraînant des modifications dans les pratiques culturales etculturelles.

Cette ouverture vers l'extérieur n'aurait pas atteint la cohésion sociale de ce peuple. D'ordre économique, elle a permis au contraire à la population maraîchère de mieux $s$ 'organiser pour produire davantage et de renforcer l'effet de corps. Elle a été aussi d'ordre ludique puisque de tout temps le marais audomarois a attiré. Ilétait le lieu de villégiature pour les Sarrazins, les citadins de Saint-Omer venus prendre l'air. Avec l'octroi des congés payés en 1936, il est devenu progressivement ce lieu de détente prisé par une clientèle régionale, pour beaucoup, de pêtcheurs.

De nos jours, seules 160 familles maraîchères effectuent encore le travail de la terre, de façon mécanisée et dans une logique économique de monospécialisation. Le maraîchage demeure toujours une réalité dans la vie économique locale car cela aété une volonté politique de le maintenir sur 800 ha. Mais le maraîcher doit partager sa place avec d'autres professionnels, touristiques par exemple. Comme le proprictaire du marais doit maintenant partager sa place avec de nouveaux habitants d'origine urbaine. Alors le mélange peut être parfois détonnant et le mariage de raison devenir conflictuel lorsque les comportements font fi des règ les d'usage inhérentes à un espace de marais.

Les enjeux sociaux se situent dorénavant dans l'acceptation du pluriel, le respect de 
Un marais, ça se gère. quelques évidences chilfrées

Sans I'intervention permanente de l'homme, un marais retourne en marécage. De plus, lorsqu'il se trouve à proximité d'une agglomération de 70 000 habitants au sein d' une région de 4 000000 d'habitants, des dysfonctionnements apparaissent.

$L^{*}$ envasement des cours d"eau s'accélère. De 1986 à 1990 , une vaste opération de restauration de rivières a extrait plus de $150000 \mathrm{~m}^{3}$ sur $12 \mathrm{~km}$. Son coût: 7 millions de FF.

Les plantes aquatiques explosent. La pollution verte se chiffre en milliers de tonnes. Un faucardeur-débrouissailleur est en fonctionnement depuis l'été 1990 pour soulager régulièrement la rivière. Son coût: 0,7 million de FF.

Et la qualité générale des eaux se dégrade. La pollution de toutes origines correspond à celle générée par une ville de 300000 habitants. Le coût de son traitement: plus de 200000 millions de

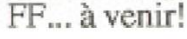

l'autre et la recherche d'un nouvel équilibre basé sur des valeurs autres.

L'identité de ce pays d'eau - lieu de travail pour les uns, lieu de détente pour les autres - demande donc à être travaillée avec finesse et perspicacité à l'heure où dame tourisme prend rang dans la vie économique locale et où les frontières s'ouvrent et aussi lorsque s'expriment quelques résistances à l'intervention publique dans la gestion collective du marais, traditionnellement assurée par ses propriétaires regroupés en association.

Depuis toujours, le paysan, le travailleur de la terre qui est aussi l'homme de pays a été vilipendé de toutes les façons par les autres classes sociales. Par les nobles d'abord, ensuite par les bourgeois et pour finir par les ouvriers des villes. Par tout le monde en somme. Quoi d'étonnant, s'il a cherché à s'evader de sa condition. (Pierre Jaker Helias)

Le marais audomarois ou le fruit d'une construction humaine fragile, durable pour combien de temps?

Imaginez qu'au 7ème siècle, la mer venait lécher les collines d'Artois situées à plus de $40 \mathrm{~km}$ des côtes actuelles. En se retirant, elle a laissé un marécage. Puis, avec le temps, la rigueur, les enseignements techniques fournis par les moines bénédictins ou plus tard recherchés auprès des Hollandais, et aussi la volonté de ces brouckailleurs, cette zone inondable s ${ }^{4}$ est transformée en une véritable mosaïque de terres et d'eau.

Cette histoire raccourcie ne dit pas les raisons pour lesquelles un chantier aussi ambitieux et échelonné sur treize siècles a été entrepris. Elle était de deux ordres:

- disposer de nouvelles terres pour nourrir la population de la ville et des environs en plein essor;

- et offrir à la ville de Saint-Omer, un accès à la mer pour le développement de ses échanges commerciaux avec la Flandre.

Selon les récits, la conquête des terres mauresques aété une aventure folle, comme elle a nourri des luttes de pouvoir et développé un esprit d'initiative et de solidarité.

En effet, ici, l'eau a été conquise, maitrisée pour être canalisée dans des chenaux crếés par l'homme et qui sont à l'origine de ce paysage particulier de la Flandre maritime actuelle. L'eau n'est plus libre de vagabonder. Mais il peut lui arriver d'avoir des sursauts et en cas de crues de rappeler que cette région est de nature inondable, car situéeà quelques petits mètres au-dessus du niveau de la mer.

Alors, les promesses techniques ont été grandes pour toujours avancer dans la lutte contre l'eau et dans la mise hors d'eau de ces terres dites hautes ou basses, porteuses de légumes pour l'hiver ou l'été.

La plus spectaculaire fut notamment celle mise en oeuvre par la technique d'assèchement dite de polérisation venue des Pays-Bas et caractérisant la partie centrale du marais, faite de terres en forme de lanières ou lègres qu'une carte topographique dévoile en première lecture.

Cette performance technique a révélé la nécessité d'une organisation de travail collective etpermanente pour rendre durable 1'oeuvre, Conscients, les propriétaires ont délégué certaines de leurs obligations à une association syndicale qui fonctionne toujours - l'époque où les pouvoirs de décisions politiques se décentralisent - sur des principes de lois particulières et datant du début XIXe siècle!

Malgré tout, une inquiétude sournoise continuait de miner ces créateurs et défenseurs de leur outil de travail et milieu de vie: l'inondation. La réponse ne peut être apportếe qu'en 1984 quand d'importants ouvrages hydrauliques réalisés dans le delta de $\mathrm{l}^{\prime} \mathrm{Aa}^{(4)}$ - région hydraulique dont dépend la petite région de Saint-Omer - sont venus rationaliser le travail de la gestion de l'eau. L'intervention publique ainsi sollicitee, s'est traduite sur les plans financier et juridique par la mise en place d'unenouvelle organisation, repositionnant par conséquence les responsabilités vers le public et les intérêts vers le général.

De même et à court terme, le travail de gestion de la rivière prise sous l'angle de l'entretien par curage et faucardage laisse présager d'une alliance forcée avec la collectivité publique. Cette situation s'explique par l'ampleur des problèmes rencontrés pour la gestion du marais situés au-delà des frontières de l'audomarois et devenus etdémesure totale avec des moyens structurels classiques.

La vie et le devenir de cet espace naturel remarquable restent donc toujours à déterminer par les aménagementsentrepris ou à entreprendre à l'amont et à l'aval. Par ses propres activités, aussi. Et là, elles ne sont pas à l'abri non plus de tout développement initié en externe - par le canal de l'Europe communautaire ou non et pouvant générer des dysfonctionnements sournois sur des espaces par essence contraignants car fragiles.

Ainsi, par nécessité économique, l'activité agricole et maraîchère se restructure et laisse des espaces disponibles propices au développement de friches, de plantations de peupliers, d'ếtangs, voired'implantations d'habitats de loisir plus ou moins organisées... et donc au développement d'une mosaïque d'usages de plus en plus difficilement gérables, y compris en termes d'image de la région.

À l'approche de l'an 2000, celle-ci pourrait bienêtre lecatalyseur pour bât ir un nouveau projet de développement économique et environnemental pour l'audomarois en réfléchissant sur les limites de l'acceptable dans l'évolution d'un site patrimonial.

\section{Le marais audomarois et son intérêt patrimonial, une nécessité vitale d'agir en partenariat pour sa préservation}

Un coup de zoom, côté Est et sans rien y comprendre, vous êtes brutalement dans une autre ambiance.

C'est le royaume de la nature pour les enfants et adultes invités à découvrir, lors d'une promenade, un héron cendré quiplane au-dessus de l'étang, des grèbes huppés en 


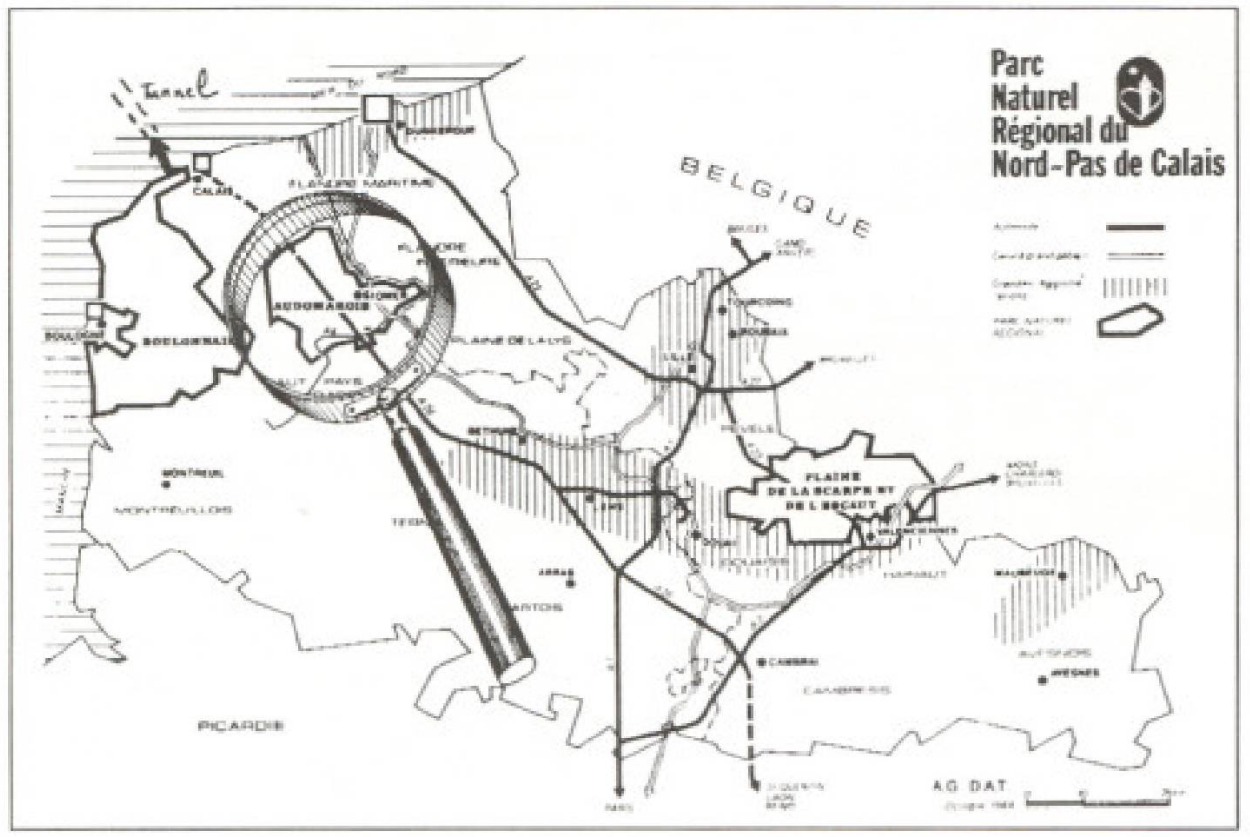

parade nuptiale, des cormorans en train de se sécher les ailes ou alors à apprécier le jeu des lumières entre la terre et l'eau ou encore à prendre le temps d'écouter le chant des oiseaux, le coassement des grenouilles.

La Grange-Nature offre ce petit moment de rêve avec ses montages audiovisuels pédagogiques. Plus de 30000 visiteurs y passent et prennent rendez-vous avec la nature située à la porte de cet équipement du Parc naturel régional qui a acheté un ancien site de tourbières géré en réserve naturelle. Dans ce marais audomarois fortement humanisé, un tel îlot de bois, roselières, prairies, étangs... étonne et dépayse.

Le maintien de cette diversité des milieux naturels qui fait la richesse paysagère et biologique du site du Romelaere nécessite d'importantes actions de gestion. Elles sont réalisées en partie par des chantiers de jeunes dans le cadre des échanges définis par le jumelage. Elles servent comme de

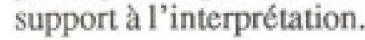

Le site du Romelaere est aussi, pour le Parc, un site de démonstration pour

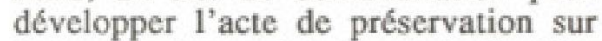
l'ensemble du marais audomarois. Certains dispositifs ont été mis en place ou se négocient pour permettre à d'autres collectivités publique dese rendre acquéreur de terrains mis en vente ou d'envisager des contrats particuliers de gestion avec des agriculteurs qui se sentent garants du patrimoine marais. Ce travail commence à porter ses fruits. Une certaine confiance se réinstalle chez divers agriculteurs herbagers.

Cela ne règle pas tout, malheureusement. II est dit dans un dépliant du Parc: "L'eau est l'élément primordial. Son niveau et sa qualité commandent la répartition des végétaux et par là des animaux".

Ce pays d'eau fait rêver, il peut faire peur à celui qui s'aventure avec une barque dans ce lacis de fossés. Il est aussi un pays qui vit de ses activités liées ou non à l'eau et qui se trouve dépendant d'un environnement extérieur lourd de consếquences. Actuellement, des enjeux importants se excédentaire mais pour une eau de qualité.

En effet, le marais doit "encaisser" régulièrement une pollution équivalente à celle d'une ville de 300000 habitants, la rançon d'un modèle de développement que les adolescents de l'audomarois récemment interviewés pour les besoins de l'étude sur 1 'image de marque de la région, rejettent.

Alors, le Parc mobilise et se mobilise pour obtenir des résultats rapides auprès de tous les acteurs économiques. Mais, pour avancer de manière constructive, il faudra y compris dans ce domaine de la gestion de la qualité de l'eau et vu le contexte français - innover, travailler et parler vrai.

Or, ce pays d'eau peut faire vivre sur ses valeurs patrimoniales ou, dans un jargon touristico-français, sur la base de produits spécifiques de tourisme-nature tels que:

- la promenade en bateau type bacôve;

- des centres d'information et d'interprétation du patrimoine qui invitent à comprendre et à découvrir les milieux naturels, l'histoire et la vie des habitants du marais; ou alors de façon plus ciblées, des jouent, vitaux: la lutte ancestrale vis-à-vis de l'eau n'est plus une lutte contre l'eau
Le développement des echanges techniques

Traditionnellement, les responsables du marais audomarois se sont tournés vers l'extérieur pour comprendre et améliorer les techniques d'aménagement et de gestion d'une zone humide, telle la poldérisation.

Quelques siecles après, Espace naturel régional poursuit la tradition par le jumelage entre trois zones humides du Nord-Ouest de 1'Europe officialisé le 5 juin 1990.

Mais cet organisme public n'en est pas à son premier coup d'essai pour instaurer des échanges techniques. Dans le domaine de la gestion de l'environnement, les milieux dunaires, notamment ceux classés en réserve naturelle d'État, sont également concernés par le réseau européen dessites jumelés: Eurosite. Dans le domaine de la pédagogie de l'environnement et nouvellement de l'interprétation du patrimoine, des relations privilégiées se sont développées avec le Québecet le CÉGEP de Saint-Félicien. Un festival européen, le premier en la matière, aété créé en 1990 (pour être renouvelé tous les deux ans) où enfants, formateurs, artisteset responsables institutionnels se sont retrouvés et ont créé. Dans cette aventure, le Québec était devenu, pour un temps, européen.

Toutes ces initiatives ont alimenté dernièrement un débat sur les moyens d'une gestion appropriée de l'environnement par et avec les responsables des réserves et parcs naturels de France, réunis dans le Nord de la France.

activiı́s ćconomiques génératrices de vie sociale et culturelle: restauration, đégustation-vente de produits locaux, spectacles liés à l'eau...

Les atouts sont là, potentiellement valorisables. La volonté d'entreprendre existe, insuffisamment contrôlée dans le cadre foncier et réglementaire qui pèse actuellement sur cette zone humide, ecrin de verdure qui ne doit pas devenir vide de contenu ni vide d'âme.

Lapréservation du site de l'audomaroisqui sous-tend des actions de gestion et de développement pour pérenniser son intérềt patrimonial, est l'affaire de tous. Mais tout est question d'cquilibre voire de mesure, à tout moment et en tout lieu. 
Une approche déclinée pour le marais

La demande touristique est là à la porte de la ville de Saint-Omer, fortement centrée sur le marais et son produit d'appel: la promenade en bateau.

Satisfaire cette demande sans compromettre l'intérêt d'une zone humide signifiait pour Espace naturel régional:

- de faire fédérer les partenaires et professionnels du tourisme pour promouvoir

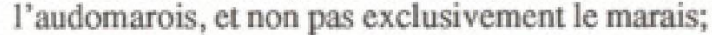

- de créer d'autres centres d'intérêt touristique, en périphérie du marais;

- de développer des centres d'information et d'interprétation du patrimoine marais, à partir de méthodes nord-américaines;

- de définir une politique d'image de marque commune à trois partenaires institutionnels oeuvrant dans le développement;

- de gérer une qualité de vie pour les habitants du marais, côté terre, côté eau;

- et aussi de faire vivre et cohabiter des populations différentes sur un site pour qu'il y ait ni rejet par la population locale, ni perte d'identité.

Plus particulièrement, en matière de tourisme fluvial, l'accent a êtế mis:

sur laclientèle de groupe, avec la miseen place de produitsciblés et le développement de forfaits à la journée ou de court séjour;

- sur le réseau régional d'infrastructures de tourisme fluvial;

- et sur des animations de type fête nautique, circuit nautique illuminé.

Et ci-après, quelques questions que suscite l'exemple du marais audomarois et qui furent posées lors d'un colloque international organisé à Tour en mars 1990 sur la politique de développement touristique de la voie d'eau:

- peut-on raisonnablement faire de la préservation d'un patrimoine quand l'initiative privée n'est pas contrôlée et quand l'espace demeure privatif, même si l'usage de la rivière est collectif (droit coutumier)?

- faut-il promouvoir, et comment, un site sur ses qualités patrimoniales quand les mesures de protection ne sont pas en plàce?

- ne faudrait-il pas envisager pour les usagers fluvieux un droit d'accès aux sites privés ouverts au public, redevance qui serait attribuée aux gestionnaires de ces sites pour faciliter la préservation et l'entretien?

- à l'image de ce qui se fait aux Pays-Bas, pourquoi ne pas envisager de faire participer les activitéséconomiques qui vivent du marais à la gestion patrimoniale, et comment en répartir le coût?

"La terre ne nous est que prêtée." (Saint-Exupéry)

\section{Un site naturel entre les mains d'espace naturel régional}

Se faire accepter pour être entendu, être à l'écoute de la population locale et la rendre participative, disposer de moyens toujours suffisants pour une préservation maximale du site, développer des actions de gestion innovatrices face au déclin progressif de l'activité agricole et maraîchère, voire face à la déresponsabilisation des gestionnaires traditionnels, mais aussi devoir allier en permanence la préservation de ces milieux naturels et leurouvertureà un public régional et européen à la recherche de détente, ainsi que travailler avec d'autres partenaires pour la mise en oeuvre d'une politique de développement local harmonieuse...

... Telle est la mission-combat à laquelle se livre Espace naturel régional chargé par le Conseil de la Région du Nord-Pas-de-Calais de gérer et d'animer le marais audomarois, cet espace protégé et intégré depuis 1986 dans le réseau des Parcs naturels de France.

Cette mission est en effet un combat permanent car elle vit sur des paradoxes.

Pour l'État, la préservation de zones humidesest un objectif poursuivi mais n'en constitue pas pour autant un axe majeur de sa politique en matière d'environnement qui balbutie toujours. Peu de moyens financiers y sont consacrés, à l'opposé de ce qui se passe de l'autre côté du Channel(s). Et l'action réglementaire est diluée.

- Pour la région, sa volonté de préserver un tel espace est clairement affichée. Elles'est traduite par la mise en place duParc naturel régional et par la mise à disposition d'un outil-conseil en environnement, Espace naturel régional, et donc par desengagements financiers conséquents. Ses moyens ne sont en aucun cas d'ordre réglementaire.

- Au niveau des communes, la politique d'éco-développement est encore pour le moins frileuse. L'environnement est toujours perçu par les élus locaux comme une gêne au développement économique qu'ils initient. Deschangements d'attitude devraient s'observer... puisque le mouvement écologique représente un poids dans la vie démocratique locale depuis les élections de 1989. Néanmoins, il reste encore quelque chemin à parcourir pour satisfaire pleinement à l'adage "Pensez globalement pour agir localement". Ceci passera aussi par la reconnaissance du métier de maire et ce, face aux responsabilités accrues et démultipliées depuis la décentralisation.

- Quant aux propriétaires de terres de marais - au nombre de 10000 beaucoup veulent se livrer à la spéculation foncière et immobilière, qui s'enflamme avec l'ouverture prochaine du lien fixe Transmancheet du marchế unique européen. Certes, la pratique locale d'un tourisme de cueillette n'aura bientôt plus droit de cité mais le mal ne fera qu'amplifier avec moins de prise réelle pour l'enrayer: les opérateurs touristiques internationaux vendront du "clef en main" en grande quantité.

Or, la protection actuelle de ce site privé à usage collectif repose sur le simple respect de documents réglementaires d'urbanisme tels que les Plans d'Occupation des Sols. Devant de tels appétits, elle n'en est que précaire puisque sur décision du conseil municipal, le document approuvé par la population peut être révisé(i)

On aurait tendance à dire que l'aménagement du territoire se fait au quotidien et se décide localement même si, ici, l'intercommunalité s'exerce depuis 1962! Celle-ci ne peut en aucune manière régler les divorces de la fiscalité locale dont les principes essentiels reposent sur la richesse de la commune calculée en fonction 
Espace naturel régional, c'est:

Né en 1978 pour appliquer la politique d'environnement du Conseil de Région du Nord-Pas-de-Calais, Espace naturel régional intervient pour:

- gérer et animer le Parc naturel régional, le seul de France à être éclaté sur plusieurs espaces géographiques;

- créer un réseau d'espaces naturels protégés;

- conserver les races animales et espèces végétales régionales;

- sensibiliser le public et surtout les jeunes à l'environnement.

C'est done un organisme publie régional de 80 personnes quifait, fédère, impulse. Il demeure un organisme au niveau français qui expérimente dans le domaine de l'environnement.

\section{Contact: Espace naturel régional Le Grand Vannage - 62510 Arques (France) \\ Tél:: 21.98 .62 .98 \\ Fax: 21.98.37.05}

de l'importance de l'urbanisation. Des systèmes de compensation pourraient être $m i s$ en place et ainsi valoriser l'action communale lorsque l'élu entend préserver les espaces naturels de toute construction. Cela supposerait d'instaurer un principe de solidarité "naturelle".

Le contrat fondateur n'est pas passé seulement par les hommes entre eux, il doit avoir la nature pour partenaire. Il n'est pas seulement naturel. (Michel Serres)

\section{Alors... alors...}

Quand des clignotants s'allument - rouges, bien sûr - il est bon de savoir s"arrêter, prendre le temps de l'analyse pour une prise de conscience collective des failles actuelles et pour la mise en place d'une politique réelle de préservation de cette zone humide du Nord-Ouest de l'Europe sans frontière. Telle est l'ambition actuellement poursuivie par Espace naturel régional qui estime par ailleurs que les investissements humains et financiers déployés depuis plus de dix ans sur ce site ne sont pas suffisamment valorisés et partagés. L'effort doit être plus incisif en direction du "privé" qui vitdu marais ou qui l'utilise pour l'exercice de son activité professionnelle. Comme la solidarité doit être aussi nationale.

Un pas vers le premier parc national français de zones humides et pour une conformité des politiques européennes en la matière? Des procédures de concertation élargie, d'expertises vont être engagées dans ce sens dès 1991.

Les mouvements d'opinion et les enjeux de l'eau sont tels que l'espoir est permis de ne pas voir le marais audomarois - cet espace de vie - condamné à être une image, voire un mirage.

\section{Notes explieativas}

(1) Bateau à voile du moven-ăge.

(2) Nouveau nom qui serait donné a la region du NordPas-de-Calais, l'une des 21 régions de France devenues collectivités territoriales par décret d'application de la loi de 1983 relative a la decentralisation et au transtert de competences entre l'Etat et les collectivites.

(3) Grande barque à fond plat, en chène, d'origine néerlandaise pouvant transporter jusqu'à cinq tonnes de chous-fleurs; barque toujours tabriqué a Saint-Omer, reconvertie progressivement pour le transport de personnes dans le cadre de visites guidées.

14) Riegian comprise entre Calais-Durkerque et SaintOmer, alimentée par l'Aa et qui, â partir de SaintOmer, se trouve canalisèe, également dénommé régiondes wateringues, itgojon du plat pays trançąais ou région de ba Flandre maritime

(5) La région des Broads, région jumelée avec le marais audomarois est devenue Parc national depuis 1909. De mente, côte Pays-Bas, De Werribben - autre zone humide jumelé avec le marais audomarois - est réserve naturelle d'État

(6) Des logiques spatiales etéconomiquesdifférentes s'affrontent donc sur un même espace naturel identifie Iréparti sur quatorze communes et ne représentant qu'un morceau de territoire communalh pour une mème logique fiscale.

Quelques róferences bibliographiques

BILLAUD, Jean-Paul, Le Marais Poitevin - rencontre de la terre et de l'eau, Édition L'Harmattan, 1984

DEVAUX, Gonzague, Lo marais audomarois propositions d'amenagement, Document de synthese elabore al partir d'etudes scientifiques nombreuses, Agence d'urbanisme de la région audomaroise, 1976.

DRUBBRULLE, Anne, Le tourisme fluvial Nord-Pas-de. Calais - un nouveau créneau pour le tourisme rüglonal, Université des sciences et techniques de Lille, 1985

Dufly. Odile, L'hóbargement touristique dans le marais audomarois - analyse du phënomène ot propositions Agenced urbanisme de la région audomaroise, 1977.

L'envirennement de la France au pied du mur - une approche globale pour des bco-pays, Actes du colloque de l'Entente européenne de Tentionnement des 3 et 4 mai 1990.

LEPERS, Pascale, Le patrimoine eulturel du marais audomarois - recherche sur les antecedents d'un milieu original en voie de disparition. Espace naturel regional, 1981.

LEPERS, Pascale Promenade dans le marais audomarols, Guide sur l'histoire do la mise en waleur de ce site et pour une invitation a la lacture de paysage. Espace naturel régional, 1985.
REVEL, Veronique, La dynamique touristique dans r'Ousst du marais audomarois, Université des sciences et techriques de Lille, 1989.

SERRES, Michel, Le eontrat naturel, Édition F. Bourin, 1990 